Literatura y Lingüística $\mathrm{N}^{\circ} 24$

ISSN 0716-5811 / pp. 183-197

\title{
El atributo de funcionalidad en el cibergénero Artículo de Investigación*
}

\author{
María Marcela Puebla** \\ Andrea Puchmuller***
}

\section{Resumen}

El propósito de este trabajo es describir y analizar el cibergénero científico-académico Artículo de Investigación desde el punto de vista de su funcionalidad y en consecuencia, el valor informativo de sus links. A tal fin, se seleccionaron 90 hiperartículos pertenecientes al campo de la Psicología, Matemática y Biología y se los analizó teniendo en cuenta los atributos de funcionalidad propuestos por M. Shepherd, C. Watters y A. Kennedy (2004). Los resultados muestran que el cibergénero Artículo de Investigación presenta características funcionales que benefician ampliamente al lector-investigador y que marcan una diferencia significativa respecto a las posibilidades que ofrecen los Artículos de Investigación publicados en medios impresos.

Palabras clave: Artículo de Investigación, funcionalidad, cibergénero, links, valor informativo

\section{The functionality attribute in the cybergenre Research Article}

\begin{abstract}
The aim of this paper is to describe and analize the functionality attribute and the consequent informative value of the links of the cybergenre Research Article. 90 hyperarticles in the field of Psychology, Mathematicas and Biology were collected for this purpose and analized following M. Shepherd, C. Watters and A. Kennedy's (2004) categorization of the functionality attribute. Results show that the Research Article cybergenre has functional characteristics that greatly benefit the readerresearcher and make an important difference regarding the possibilities offered by paper issued Research Articles.
\end{abstract}

Key words: Research Article, functionality, cybergenre, links, informative value

Recibido: 11-04-2011 Aceptado: 04-05-2011

* El presente trabajo se realizó en el marco de la Línea 4: La literacidad digital en el ámbito académico-científico del proyecto "Desarrollo de la competencia de género para la comunicación académico-científica en un contexto plurilingüe".

** Profesora y Traductora de Inglés. Especialista en Educación Superior. Universidad Nacional de San Luis.mpuebla@unsl.edu.ar o mm.puebla@gmail.com

*** Licenciada en Lengua y Literatura Inglesa. Universidad Nacional de San Luis. Instituto de Formación Docente de San Luis. puchmuller@yahoo.com.ar 
El atributo de funcionalidad en el cibergénero. Artículo de Investigación / María Marcela Puebla y Andrea Puchmuller

\section{Introducción}

La presente investigación se enmarca en el proyecto "Desarrollo de la competencia de género para la comunicación académico-científica en un contexto plurilingüe" pero toma como punto de partida datos recogidos en el PROICO 4-1-0403 recientemente concluido. Estos datos surgen de Encuestas realizadas a los docentes-investigadores Titulares de las Facultades de Ciencias Humanas, de Química, Bioquímica y Farmacia y de Ciencias Físico-Matemáticas y Naturales de la Universidad Nacional de San Luis (UNSL) ${ }^{2}$, las cuales tuvieron por objetivo conocer qué géneros utilizan estos docentes para su formación como profesionales, en qué idiomas leen, si consultan Internet, qué Instrumentos de Evaluación utilizan, qué géneros sugieren a sus alumnos que lean, entre otros. De los datos recogidos en estas encuestas se tomaron dos aspectos que consideramos relevantes para nuestro estudio: el primero es que, en todas las carreras mencionadas con anterioridad, aproximadamente un 85\% de profesores lee Artículos de Investigación (AI) escritos en Español publicados en medios impresos (un 45\% lee Artículos de Investigación escritos en Inglés), y el segundo aspecto es que un 90\% de docentes consulta Internet en busca de AI publicados en sitios de otras universidades del mundo o journals on-line. En ambos casos el principal propósito de lectura es el de (in)formación profesional.

Dado que los géneros académico-científicos constituyen en el ámbito universitario el medio por excelencia a través del cual los docentes actualizan sus saberes (Artículos de Divulgación, Artículos de Investigación, Conferencias, Reviews) y a partir de la conjunción de los datos anteriormente mencionados surge la inquietud de indagar qué beneficios brindaría al docente-investigador la lectura de Ciber-Artículos de Investigación escritos en Inglés ya que en la Web se suma a la caracterización tradicional de géneros < contenido, forma $>$ la característica distintiva de los cibergéneros < contenido, forma, funcionalidad> (Shepherd \& Watters, 1999). En consecuencia, el propósito de este trabajo es describir y analizar el cibergénero científico-académico Artículo de Investigación desde el punto de vista de su funcionalidad. Teniendo en cuenta que el atributo de "...funcionalidad refiere a las capacidades disponibles en el nuevo medio" (Shepherd \& Watters, 1999) y esta investigación se centró, por un lado, en la evaluación de la presencia de links en las

1 Pedagogía de la lengua basada en géneros. Desarrollo de materiales para la comprensiónproducción de textos en la comunidad universitaria.

2 La encuesta no se realizó en la Facultad de Ingenieria y Cs. Económico-Sociales, con sede en la ciudad de Villa Mercedes. 
páginas en las que se encontraban los artículos de investigación y en los artículos mismos y, por el otro, en la calidad de la información que éstos proporcionaban.

\section{Marco Teórico: Los géneros digitales}

La palabra "género" proviene del latín genus y era utilizada en la esfera de la filosofía clásica para la clasificación (Breure, 2001). En la actualidad, es también utilizada como una manera de clasificar estilos o tipos de textos que aparecen en los medios a través de la identificación de elementos que éstos tengan en común. Este concepto se desarrolló particularmente en el estudio del cine, la literatura y las artes y, con el correr de los años, ha sido ampliamente utilizado en el campo de los medios de comunicación con el mismo fin, el de clasificar.

"La migración hacia Internet es continua e irreversible" (Cassany) ${ }^{3}$ y exige una necesaria democratización de las posibilidades que ofrece la Web para lograr una inserción en los distintos ámbitos académicocientíficos. Crowston y Williams (en Breure, 2001) fueron uno de los primeros investigadores en ver la importancia del concepto de género para analizar la comunicación e información en la Internet, pero Shepherd y Watters (en Breure, 2001) inventaron el término "cibergénero" para referirse a los géneros digitales; es decir, aquellos encontrados en la World Wide Web, a partir de la cual se ha producido una continua aparición y consecuente diversidad de cibergéneros. En la WWW podemos encontrar géneros que son idénticos a géneros ya existentes en otros medios, es decir, géneros que se han trasladado al medio digital sin explotar las posibilidades que éste ofrece; cibergéneros que han evolucionado a partir de éstos agregando ciertas características de funcionalidad propias del ciberespacio como, por ejemplo, los links; o podemos encontrar cibergéneros propiamente dichos, es decir, aquellos que han surgido de manera espontánea en la Web y fuera de la cual no podrían existir. En otras palabras, y tal como lo indica la Figura 1, los cibergéneros se clasifican en dos clases de subgéneros: los 'ya existentes' (diarios, biografías, diccionarios, etc.) y los 'nuevos' (hiperficción, home pages, juegos, etc.) (Shepherd \& Watters, en Breure, 2001).

3 Cassany, Daniel. Investigación y propuestas sobre literacidad actual: multiliteracidad, Internet y criticidad. Cátedra UNESCO para la lectura y la escritura. Universidad de Concepción, Chile. Disponible en www.udsec.cl/catedraunesco/CASSANY.pdf 
El atributo de funcionalidad en el cibergénero. Artículo de Investigación / María Marcela Puebla y Andrea Puchmuller

Figura 1. La evolución de los cibergéneros (Shepherd \& Watters, en Breure 2001)

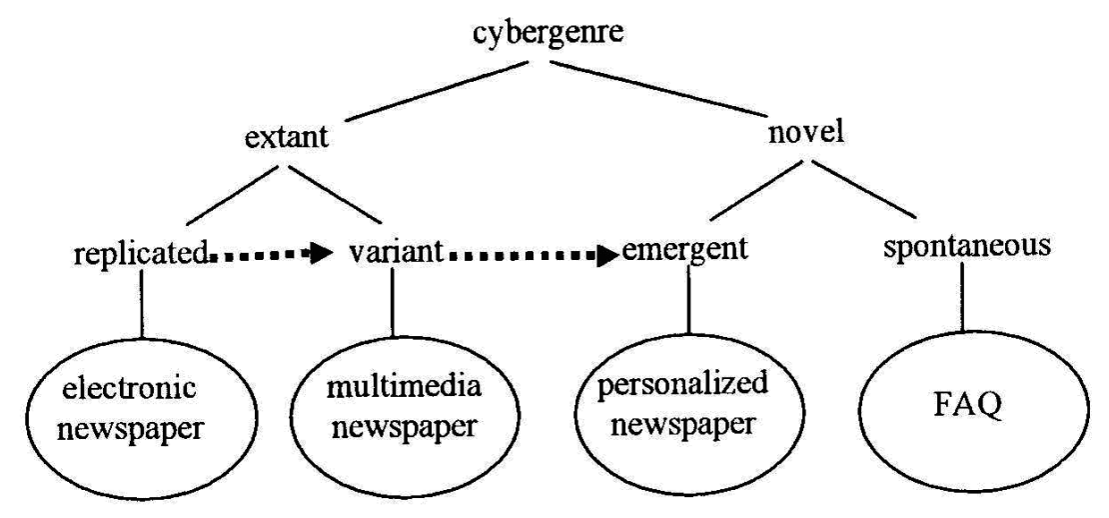

La inserción de un link en un texto a partir de la selección de un ítem lexical posibilita la interconexión de elementos textuales, organización de y acceso a la información, provocando, al mismo tiempo, la evolución del género y la transformación del texto en un hipertexto.

El hipertexto no es un formato sin precedentes, en un libro, las notas a pie de página, llamados con asteriscos o notas al final del capítulo también desvían la atención del lector hacia otras fuentes o puntos de vista, creando así un hipertexto cognitivo. Sin embargo, en un hipertexto computacional tales relaciones existen físicamente, ya que "...los hipertextos son textos o elementos textuales interconectados por links electrónicos (Engebretsen, 2000, p. 4).

\section{El atributo de funcionalidad}

La creciente digitalización de la información y el predominio de Internet en los años '90 ha extendido el estudio de los géneros en medios no-digitales a los géneros digitales. Los géneros que se encuentran en medios no computacionales se caracterizan comúnmente por la dupla $<$ contenido, forma > o < propósito, forma $>$ en las que contenido refiere a los temas desarrollados, el propósito refiere al propósito comunicativo socialmente reconocido y la forma incluye tanto el medio de comunicación como las características lingüísticas y estructurales (Yates \& Orlikowski, en Shepherd \& Watters, 1999).

Los géneros digitales están caracterizados por su <contenido, forma, funcionalidad> (Breure, 2001) por lo tanto, un tema central en el estudio de los mismos es el de la funcionalidad. Sheperd \& Watters (1999, p. 1) definen a la funcionalidad como "las capacidades disponi-

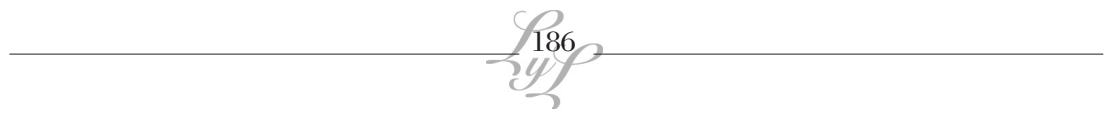


bles en el nuevo medio" que no han existido en otros medios y que, en consecuencia, han provocado la evolución de los géneros implicando una manera de interactuar con ellos, así como también, de despertar expectativas con respecto a los mismos. El atributo de funcionalidad, propio de los hipertextos, está dado principalmente por el input visual, la interactividad y los links (Shepherd, Watters \& Kennedy, 2004). Por lo tanto, y conforme a lo expuesto por Shepherd, Watters y Kennedy (2004), para determinar la funcionalidad es necesario tener en cuenta las siguientes características:

Número de links en la página Web.

Número de E-mail Links.

Proporción de links navigacionales a otros links en otras páginas Web dentro del mismo sitio.

Proporción de links dentro de la misma página.

Proporción de links a otras páginas en otros sitios.

Inclusión de JavaScript de otro archivo externo.

Escritura de JavaScript HTML.

Presencia de formularios.

Número de entradas en el formulario.

Existencia del primer tag ${ }^{4}$ como un tag de ejecución

\section{El valor informativo de los links}

Los links son elementos de la retórica que conducen al lector de un texto a otro texto y crean significados por sí mismos. Existen distintas taxonomías de links (Lewis, et al., en Harrison, 2002; Harrison, 2002; Puchmüller \& Puebla, 2006), las cuales sugieren maneras diversas de relacionar los elementos que unen (Burbules \& Callister, 2001). Claire Harrison (2002) establece una diferencia entre el valor organizativo y el valor informativo de los links. Con respecto al valor organizativo distingue entre links "especialmente creados" (strongly authored); es decir, escritos con un propósito y links "de conexión a otra página" (weakly authored); es decir, creados a través de modelos computacionales. Con respecto al valor informativo esta autora sugiere la siguiente taxonomía:

a-links de autorización (authorizing links): permiten el acceso a información formal y legal de autoridades de una organización, lo cual contribuye a la autenticación de un sitio;

4 Tag puede traducirse como rótulo o instrucción, pero se ha optado por dejar el término en inglés, ya que es de la manera en que este término se emplea en los lenguajes de programación. 
El atributo de funcionalidad en el cibergénero. Artículo de Investigación / María Marcela

Puebla y Andrea Puchmuller

b-links de comentarios (commenting links): proporcionan opiniones acerca del sitio y de su contenido;

c-links de ampliación de la información (enhancing links): ofrecen mayores detalles o amplían la información objetiva sobre los contenidos del sitio;

d-links de ejemplificación (exemplifying link): proporcionan ejemplos específicos de contenido dentro de una categoría más amplia;

e-links de cambio-de-modo (mode-changing links): llevan a que el usuario alterne, por ejemplo, entre la lectura de un texto y la lectura de un gráfico o la visualización de un video;

f-links de referencias/citas (referencing/citing links: contienen información que complementa el contenido del sitio;

g-links de auto-selección (self-selecting links): permiten que el usuario acote su búsqueda realizando elecciones que van a depender de su edad, sexo, ubicación geográfica, intereses personales, etc. (p. 10):

\section{Metodología}

Para la realización de esta investigación se siguió una lógica de tipo cuantitativo. El mismo permitió abordar al objeto de estudio (la funcionalidad en hiperartículos de investigación) desde una doble perspectiva, es decir, determinar la funcionalidad de los Artículos de Investigación a partir de la presencia de links y el valor informativo de los mismos. Se tomó como estrategia de investigación un diseño de análisis de contenido. El mismo se propuso contrastar teoría (categorías teóricas ut supra) con los hipertextos como fuente de encuentro con la empiria.

Para la conformación de la muestra se seleccionaron 90 hiperartículos pertenecientes al cibergénero Artículo de Investigación que, de acuerdo a la categorización de páginas Web descripta por Shepherd, Watters y Kennedy (2004), pertenecen a "sitios de recursos" (Resources) cuyo propósito principal es el de proporcionar información sobre la naturaleza de un tema o recurso dado. La funcionalidad principal de estos sitios incluye discusión, browsing, dirección de e-mail, interacción y capacidad de búsqueda en ese sitio.

Los 90 hiperartículos se seleccionaron teniendo en cuenta que presentaran un Abstract e Introducción, Método, Resultados y Discusión respondiendo a la estructura del Artículo de Investigación descripta por Swales (1990). Los artículos del corpus pertenecen al campo de la Psico- 
logía, Matemática y Biología (30 en cada caso) a fin de que representaran a una ciencia perteneciente a cada una de las Facultades de la Universidad Nacional de San Luis anteriormente mencionadas. Otro criterio de selección de los hipertextos para la formación del corpus, consistió en tipear en el buscador de "Google" el campo científico seleccionado (Psicología, Biología y Matemática) más el género a investigar: "Artículos de Investigación", ambos en el idioma inglés ${ }^{5}$. De la lista proporcionada por el buscador, se seleccionaron aquellos hipertextos que cumplieran con las características del cibergénero mencionado y que fueran de libre acceso. Los mismos se analizaron teniendo en cuenta las categorías de funcionalidad propuestas por M. Shepherd, C. Watters y A. Kennedy (2004) que pudieran responder al interés de la investigación, es decir, aquellas que fueran de utilidad para la lectura, formación y actualización del docente-investigador. Las otras características no incluidas están relacionadas con el diseño y algunos aspectos técnicos del hipertexto. Asimismo, se acotó la primera característica al género específico Artículo de Investigación.

Como técnica de recolección y sistematización de datos se construyeron dos instrumentos (cuadros de doble entrada), que permitieron descomponer a los hipertextos y a los links, de modo tal que emergieran los aspectos que se buscaba estudiar. El primer instrumento permitió observar si las páginas en las que se encontraban los artículos de investigación y los artículos mismos habían incorporado una de las características de funcionalidad, a saber, el link. Las categorías tenidas en cuenta para el análisis surgen de una redefinición de las características detalladas por Shepherd, Watters y Kennedy (2004). Esta redefinición se realizó en base al propósito del estudio y son las siguientes:

-Porcentaje de Hiper-artículos de Investigación ${ }^{6}$ que contienen links.

-Porcentaje de Hiper-artículos de Investigación que contienen E-mail links.

-Proporción de Hiper-artículos de Investigación que contienen links navigacionales a otros links en otras páginas Web dentro del mismo sitio.

-Proporción de Hiper-artículos de Investigación que contienen links a ubicaciones dentro de la misma página.

-Proporción de Hiper-artículos de Investigación que contienen links a otras páginas en otros sitios.

5 Por ejemplo: Biology + research articles.

6 Para el propósito de este trabajo el Hiperartículo de Investigación constituye el primer nivel hipertextual y las páginas a las que conducen sus links, el segundo nivel. 
El atributo de funcionalidad en el cibergénero. Artículo de Investigación / María Marcela Puebla y Andrea Puchmuller

El segundo instrumento posibilitó la clasificación de estos links de acuerdo con su contenido (valor informativo) a partir de la taxonomía propuesta por Harrison (2002):

- Links de autorización (authorizing links)

- Links de comentarios (commenting links)

- Links de ampliación de la información (enhancing links)

- Links de ejemplificación (exemplifying link)

- Links de cambio-de-modo (mode-changing links)

- Links de referencias/citas (referencing/citing links)

- Links de auto-selección (self-selecting links)

\section{Resultados}

\section{Porcentaje de Hiperartículos de Investigación que contienen links}

Un $84 \%$ de los hiperartículos del corpus $(76)^{7}$ presenta un número significativo de links en el cuerpo del trabajo, entre 5 y 40 aproximadamente. El 16\% restante (14) corresponde a artículos presentados en formato PDF que no admite la inclusión de hipervínculos. La principal característica de los links contenidos en los hiperartículos es que éstos se incorporan a partir de palabras que no pertenecen a cadenas lexicales que determinan el tema del artículo ${ }^{8}$. Estos links se insertan a partir de: 1- el nombre de el/los autor/es que remiten a otros artículos escritos por el mismo autor, 2- las citas que remiten a la sección referencias, 3- palabras tales como 'Tabla', 'Figura', 'Gráfico' y 'Video' que conducen al hiperlector a la visualización de estos elementos, y 4- las referencias bibliográficas, en las que los links se insertan a partir del nombre de los autores, del nombre del trabajo, del título de la publicación o del buscador "Google Académico", y que remiten a otras páginas o sitios en los que pueden encontrarse los artículos, o en su defecto, un link de suscripción para obtenerlos. Los hiperartículos de Matemática (22\%) constituyen la excepción ya que sus links se insertan a partir de palabras pertenecientes a las cadenas lexicales que determinan el tema del texto;

729 de psicología, 17 de matemáticas y 30 de biología.

8 Esto no ocurre en otros cibergéneros, por ejemplo, en las biografías en las que los links intertextuales se constituyen a partir de ítems lexicales que forman parte de las cadenas que determinan el tema (Cfr. Puebla, M. y Puchmuller, A. 2006).

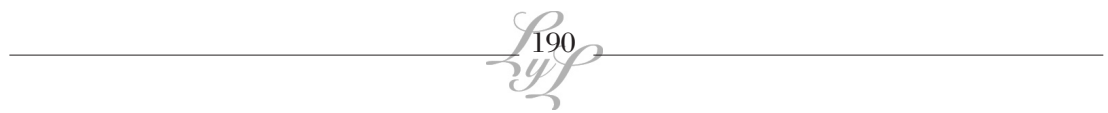


por ejemplo, un $40 \%$ de las palabras clave de los 'Abstracts' son links y remiten a artículos de investigación relacionados con el tema.

\section{Porcentaje de Hiperartículos de Investigación que contienen E-mail links}

De los hiperartículos analizados que contienen links, un 80\% (61 artículos) presenta algún tipo de link de correo electrónico. Las categorías encontradas son: "'E-mail alert" o "Subscribe to journal" que invitan al lector a suscribirse a la revista, el ícono $\nabla$ al lado del nombre del autor que permite contactarlo, "e-mail this abstract", "recommend" y "share this", los cuales permiten enviar y compartir el abstract y/o el artículo con otras personas. Un 20\% de los hiperartículos de matemáticas contiene un link llamado "erratum alert" en el cual el lector ingresa sus datos para que se le envíe por e-mail alguna corrección, modificación o actualización del artículo si la hubiere.

\section{Proporción de Hiperartículos de Investigación que contienen links navigacionales a otros links en otras páginas Web dentro del mismo sitio}

El 100\% de los hiperartículos con links (76) presenta hipervínculos que vinculan el primer nivel hipertextual con un segundo nivel perteneciente al mismo sitio. En esta categoría se encuentran links que conectan al lector con la ficha de suscripción al Journal, los datos bibliográficos necesarios para citar el artículo, un código que permite al investigador insertar el artículo como link en su blog, página web, otro artículo de su autoría o en clases on-line.

Tal como se dijo anteriormente, los links a partir de las citas en el cuerpo del trabajo y en las Referencias Bibliográficas remiten a otros hiperartículos, generalmente publicados en el mismo sitio. También se encontraron links que surgen a partir del volumen de la revista y de la fecha de publicación que llevan a una lista de todos los hiperartículos publicados por el Journal en ese volumen o en ese año.

\section{Proporción de Hiperartículos de Investigación que contienen links a ubicaciones dentro de la misma página}

Al igual que en la categoría anteriormente descripta, la totalidad de los hiperatículos de la muestra que contienen links (76), presenta hiper- 
El atributo de funcionalidad en el cibergénero. Artículo de Investigación / María Marcela

Puebla y Andrea Puchmuller

vínculos que permiten navegar en dichos artículos con mayor facilidad y rapidez. Aparecen nodos que posibilitan el rápido acceso a distintas secciones del artículo o permiten la visualización de Tablas, Figuras, Gráficos o Videos. Sin embargo, sólo un 15\% de los hiperartículos (11 artículos) analizados en relación a la presente categoría presenta un esquema de contenido de la estructura del artículo en el que todas las categorías constituyen un link, facilitando al lector el rápido acceso a cada una de sus partes.

\section{Proporción de Hiperartículos de Investigación que contienen links a otras páginas de otros sitios}

Los 76 hiperartículos de la muestra presentan links que los vinculan a otros sitios. En la sección Referencias, por ejemplo, aparecen links que remiten al buscador "Google académico" que permite buscar el artículo citado u otros relacionados, o llevan al lector a navegar otros sitios web en los que pueden consultarse artículos relacionados. Otro link perteneciente a esta categoría es el "Research Toolkit" que es un kit de herramientas que provee al lector browsers que le permiten, no solamente navegar otros sitios Web, sino también pegar y guardar citas, referencias y direcciones de la web y tomar notas mientras va leyendo el artículo.

La siguiente tabla presenta los resultados mencionados:

Tabla 1. Número de Hiperartículos de Investigación con y sin links, y sus particularidades

\begin{tabular}{|c|c|c|c|c|}
\hline & Sin Links & (14 artículos) & Formato PDF & \\
\hline & & & Particularidades & $\begin{array}{l}\text { Número de } \\
\text { Hiperartículos }\end{array}$ \\
\hline Hiperartículos & & & $\begin{array}{l}\text { Inserción de links a } \\
\text { partir de ítems lexicales } \\
\text { pertenecientes a las cadenas } \\
\text { que determinan el tema del } \\
\text { texto. }\end{array}$ & 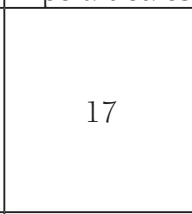 \\
\hline $\begin{array}{c}\text { de } \\
\text { Investigación }\end{array}$ & Con Links & (76 artículos) & $\begin{array}{l}\text { Presencia de algún tipo de } \\
\text { link de correo electrónico. }\end{array}$ & 61 \\
\hline (90 artículos) & & & $\begin{array}{l}\text { Presencia de links } \\
\text { navigacionales a otros links } \\
\text { dentro del mismo sitio } \\
\end{array}$ & 76 \\
\hline & & & $\begin{array}{l}\text { Inclusión de links a } \\
\text { ubicaciones dentro de la } \\
\text { misma página }\end{array}$ & 76 \\
\hline & & & $\begin{array}{l}\text { Existencia de links a otras } \\
\text { páginas de otros sitios }\end{array}$ & 76 \\
\hline
\end{tabular}




\section{Valor informativo de los links}

Con respecto al valor informativo de los links de los Hiperartículos de Investigación analizados, se observó que:

- Todos los hiperartículos contienen links de autorización, lo que otorga autenticidad y credibilidad a los mismos. En todos aparecen links que remiten al/los autor/es, y al journal o entidad responsable de la publicación del artículo.

- Sólo un 28\% de los hiperartículos (21) presenta links que cambian el modo de lectura y del procesamiento de la información. El modo principal es el de la lectura lineal del texto, pero la incorporación de links a tablas, gráficos, figuras o videos, como en el caso de los hiperartículos de biología, obligan al lector a cambiar la estrategia de lectura.

- El 100\% de los hiperartículos del corpus contiene "links de referencias/citas", siendo esta categoría una de las más frecuentes y numerosas en cuanto a su proporción, ya que cada hiperartículo contiene de 10 a 20 links de referencias. Esta categoría de links cumple, al mismo tiempo, el rol de "links de ampliación de la información", dado que también ofrecen información relacionada al tema del hiperartículo.

- Un 47\% de los hiperartículos (36) presenta links de auto-selección que permiten al hiperlector acotar o extender su búsqueda por medio de elecciones relacionadas a su condición de docente-investigador.

- La categoría de links de comentarios no aparece en este tipo de hipertextos. Sin embargo, dentro de este grupo podrían considerarse los e-mail links que permiten contactar al autor del artículo, ya que es el lector quien realiza la opción de escribir a partir de lo leído.

- Tampoco se incluyen links de ejemplificación y de ampliación de la información en los hiperartículos de investigación estudiados. Cuando se proporcionan ejemplos, éstos se encuentran en el desarrollo del hiperartículo del primer nivel hipertextual ${ }^{9}$.

9 En los artículos de Divulgación Científica los links de ejemplificación y de ampliación de la información son insertados en el cuerpo del hipertexto, ya que el propósito comunicativo del género es comunicar hallazgos científicos al público en general (Puebla \& Puchmüller, 2010). 
El atributo de funcionalidad en el cibergénero. Artículo de Investigación / María Marcela Puebla y Andrea Puchmuller

Tabla 2. Porcentajes de Hiperartículos de Investigación y tipos de links que contienen

\begin{tabular}{|c|l|}
\hline \multicolumn{2}{|c|}{$\begin{array}{c}\text { VALOR INFORMATIVO DE LOS LINKS EN LOS HIPERARTÍCULOS DE } \\
\text { INVESTIGACIÓN }\end{array}$} \\
\hline PORCENTAJE DE HIPERARTÍCULOS & TIPO DE LINKS \\
\hline $100 \%$ & Links de autorización \\
\hline $28 \%$ & Links de cambio de modo \\
\hline $100 \%$ & Links de referencias o citas \\
\hline $47 \%$ & Links de auto-selección \\
\hline $0 \%$ & Links de comentarios \\
\hline $0 \%$ & Links de ejemplificación \\
\hline $0 \%$ & Links de ampliación de la información \\
\hline
\end{tabular}

\section{Conclusiones}

En el hiper-artículo de investigación la relación entre el escritor, el lector y el conocimiento no sólo es de naturaleza cognitiva sino que también adquiere una connotación física propiamente dicha (dada en el espacio virtual). En este trabajo se observó que la interactividad que presentan los hiper-artículos de investigación está fuertemente marcada por la presencia de e-mail links que permiten al lector-investigador el contacto directo con el autor del artículo u otros miembros de su comunidad científica, permitiendo por ejemplo, "compartir" el artículo, o con la editorial, por medio de links que permiten al usuario suscribirse al Journal. Esto indicaría un cambio rotundo en la difusión del conocimiento caracterizado por la inmediatez en la transmisión de la información y la ubicuidad del investigador-autor.

Otra característica de los hiper-artículos de investigación es la gran cantidad de redes de información que se establecen a partir de la incorporación de links. En el corpus analizado se observó que estos hiperartículos adicionan información relacionada al tema que desarrollan por medio de links navigacionales a otras páginas Web dentro del mismo sitio o a otras páginas de otros sitios. La permanente conexión on line a otros artículos del mismo tema, del mismo autor u otros autores, del mismo journal u otros journals, establece innumerables redes de comunicación, información y transmisión de conocimientos que permiten transponer las barreras del artículo en si y convertir el propósito de este cibergénero en objetivos múltiples.

Desde el punto de vista de los links que aparecen en el cuerpo de los hiper-artículos de investigación, se observó que los mismos no forman parte de las cadenas lexicales que determinan el tema del artículo. Estos links intertextuales se insertan a partir de ítems lexicales formales tales 
como "nombre del autor", "tabla", "figura", "gráfico", "abstract", "referencias", etc. Esto podría deberse a que, tanto el autor como el destinatario del género, tienen una relación de igualdad, es decir, tienen el mismo nivel de conocimiento.

Con respecto al valor informativo de los links de este cibergénero se observó que los links más frecuentes son los de autorización y los de referencias/citas, ya que este tipo de links aportan valor al constructo de credibilidad del hiperartículo y del sitio que lo contiene. Los links de autorización y de referencias permiten al docente-investigador realizar juicios de valor respecto a la confiabilidad del sitio y/o hiperartículo, ya que aportan datos sobre la vigencia, actualización, y seriedad de la información y de la institución u organismo responsable de la misma. Del mismo modo, los links de ampliación de la información aparecen en la totalidad de la muestra convirtiéndose en una característica esencial del cibergénero en cuestión. En un menor porcentaje se encuentran los links de cambio de modo y los de auto-selección. Los links de cambio de modo representan una de las características más novedosas de la naturaleza de los hipertextos, ya que posibilitan que el usuario tenga acceso a datos presentados en diversas formas y utilice diferentes estrategias de procesamiento de la información. Se observa que el contenido de este tipo de links en el hiperartículo de investigación remite a tablas, figuras y gráficos, y solo en el caso de muy pocos hiperartículos de biología, a videos. Es decir, habría una cierta tendencia en este cibergénero de establecer relaciones visuales-verbales por medio de estos links, aunque el contenido de los mismos continúa siendo de naturaleza estática y no multimedial (a excepción de los videos). Asimismo, los links de autoselección no constituyen una característica definitoria del cibergénero, ya que el usuario del mismo está claramente definido y acotado: docente universitario-investigador, y por lo tanto las opciones de conexiones que se presentan no contemplan a otro tipo de hiperlector. Por último, los links de comentarios, que en otros cibergéneros ${ }^{10}$ permiten al usuario escribir sus propias opiniones acerca de un sitio, página o tema, están limitados en este cibergénero a los e-mail links que permiten el contacto con el autor.

Por lo tanto, el cibergénero artículo de investigación en la muestra analizada beneficiaría al docente-lector en tanto posibilita:

- Interactividad de naturaleza cognitiva y físico-virtual entre el autor, el lector y la información;

10 Por ejemplo en blogs, sitios de video, sitios comerciales, etc. 
El atributo de funcionalidad en el cibergénero. Artículo de Investigación / María Marcela

Puebla y Andrea Puchmuller

- Inmediatez en la transmisión del conocimiento, y el carácter de ubicuidad del autor-investigador;

- Acceso a gran cantidad de redes no sólo de información, sino también de comunicación y transmisión de conocimientos que se establecen por medio de links de referencias y citas;

- Funcionalidad dada por la presencia de links de referencias, citas, autorización, cambio de modo y auto-selección.

De lo anteriormente expuesto se concluye que el género hiperartículo de Investigación en el corpus estudiado mantiene la estructura descripta por Swales (1990) para artículos de investigación en medios analógicos. Sin embargo, al trasladarse al ciberespacio incorpora características propias del medio digital como la funcionalidad. De acuerdo al esquema propuesto por Shepperd y Watters (en Breure, 2001), el artículo de investigación surgiría en la Web como un género "existente replicado". El estudio del corpus indicaría que, al incorporar "links", los hiperartículos de investigación se ubicarían en la categoría de género "existente variante".

\section{Bibliografía}

Breure, L. (2001). Development of the Genre Concept. University of Utrecht, Department of Information and Computing Sciences [on line]. Available in: http://www.cs.uu.nl/people/ leen/GenreDev/GenreDevelopment.htm.

Burbules, N \& Callister, T. (2001). Educación: Riesgos y Promesas de las Nuevas Tecnologías de la Información. Buenos Aires. Ediciones Garnica.

Crowston, K. \& Williams, M. (1998). Reproduced and emergent genres of communication on the World-Wide Web (on line). Available in: http://crowston.syr.edu/papers/genresjournal.html.

Engebretsen, M. (2000). Hypernews and Coherence. Journal of Digital Information, Vol. 1 (7).

Harrison, C. (2002). Hypertext Links: Whither Thou Goest, and Why. First Monday. Peer Reviewed Journal on the Internet. ISSN 1396-0466. University of Illinois at Chicago Library. Recuperado el: 9 de octubre, 2006.

Puchmüller, A. \& Puebla, M. (2006). Relaciones lexicales Inter e intranodales

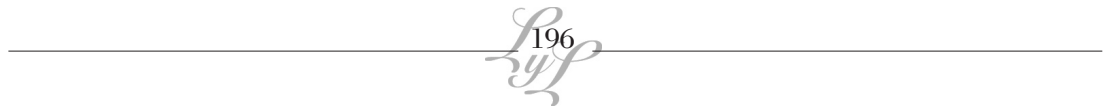


del género biografía en el Hipetexto Computacional. Revistra Claritas, Pontifícia Universidade Católica de São Paulo. N¹2 (2), 109-122. ISSN 1414-5243.

Shepherd, M. \& Watters, C. (1999). The Functionality Attribute of Cybergenres. Proceedings of the $32^{\text {nd }}$. Hawaii International Conference on System Sciences. Rinton Press.

Shepherd, M., Watters, C \& Kennedy, A. (2004). Cybergenre: Automatic Identification of Home Pages on the Web. Journal of Web Engineering, Vol. 3 No3 \& No4, 236-251.

Swales, J. (1990). Genre Analysis: English in Academic and Research Setting. Glasgow: CUP.

\section{Nota:}

Los 30 hiperartículos de psicología se extrajeron de los sitios:

"Articles, Research and Resources in Psychology" (http://kspope.com/index.php), "ScienceDirect" (http://sciencedirect.com) y "Health Care Industry" (http://articles.findarticles.com) y, en todos los casos, todos los artículos presentaban un link que proporcionaba información sobre el autor.

Los 30 hiperartículos de matemáticas se seleccionaron de los sitios:

"American Journal of Mathematics" (http://muse.jhu. edu/journals/american_journal_of_mathematics/toc/ ajm130.1.html), "Glasgow Mathematical Journal" (http://journals.cambridge.org/action/displayFulltex t type $=8 \&$ fid $=2888420 \&$ jid $=$ GMJ\&volumeId $=51 \& \mathrm{i}$ ssueId=01\&aid=2888416), y "Society for Industrial and Applied Mathematics" (http://epubs.siam.org/ SIAP/siap_toc.html).

Los 30 hiperartículos de biología corresponden al sitio: "Journal of Biology" (http://jbiol.com). 